\title{
MicroRNASeq Target Analysis and Validation by Real-Time PCR in Abdominal Aortic Aneurysm
}

Willa Sasso', Leni Moldovan², Michael Murphy²

${ }^{1}$ Indiana University School of Medicine; ${ }^{2}$ Department of Surgery, Indiana University School of Medicine

Background/ Objective: Abdominal aortic aneurysm (AAA) is an epigenetic event characterized by chronic inflammation and degeneration of the aortic wall leading to catastrophic rupture. Cigarette smoke exposure is the greatest environmental risk factor associated with AAA development. MicroRNAs (miRNA) regulate gene expression and may play a role in smoking-induced aortic inflammation. Epigenetic changes could include dysregulation of miRNA, causing post-transcriptional abnormalities pathogenic to AAA.

Methods: miRNA was extracted from plasma of 24 AAA patients and 7 risk factor matched (RFM) patients and analyzed by RNA sequencing. We compared previous (PS) and current smokers (CS) within and between both patient cohorts. Differential expression of miRNAs was analyzed by ANOVA ( $p \leq 0.05$ ). Potential targets of significant differentially expressed miRNAs were predicted using cross-analysis of TargetScan and miRanda databases.

Results: Analysis revealed 7 significantly different miRNAs between AAA CS and AAA PS and 6 significantly different miRNAs between RFM CS and RFM PS. Of greatest significance, hsamiR-223-3p was significantly downregulated as an effect of smoking cessation in AAA PS compared to AAA CS ( $p=0.000263$ ), while also showing clinically relevant expression levels. Target genes of hsa-miR-223-3p include pro-inflammatory factors IL-6, TNFa, TGF $\beta$, and MCP1. Speculatively, as tissue levels of miR-223 tend to inversely correlate with plasma levels, we could hypothesize that the observed plasma upregulation of hsa-miR-223-3p in AAA CS contributes to the pro-inflammatory microenvironment of aortic tissue.

Conclusion: Cigarette smoke contributes to epigenetic changes impacting factors of immune regulation or inflammation, eventually leading to disease states such as AAA. Inflammatoryrelated hsa-miR-223-3p is upregulated in AAA CS, suggesting its potential role in the disease course. 
Implications: Upregulation of hsa-miR-223-3p in AAA CS offers a link between disease state and the number one environmental factor attributed to AAA. This signature miRNA could serve as a biomarker for AAA or as a potential therapy target. 